\title{
Expression of phosphatase and tensin homolog and programmed cell death ligand 1 in adenosquamous carcinoma of the lung
}

\author{
Aung Myo Hlaing a , Bungo Furusato ${ }^{\text {a, b, * }}$, Emiko Udo a, b, Yuka Kitamura ${ }^{\text {a }}$, \\ Masakazu Souda ${ }^{\mathrm{a}}$, Mitsuko Masutani ${ }^{\mathrm{c}}$, Junya Fukuoka ${ }^{\mathrm{a}}$ \\ a Department of Pathology, Nagasaki University Graduate School of Biomedical Sciences, 1-7-1 Sakamoto, Nagasaki, Nagasaki, 852-8501, Japan \\ b Division of Cancer Genomics, Genomic Medical Center, Nagasaki University Hospital, 1-7-1 Sakamoto, Nagasaki, Nagasaki, 852-8501, Japan \\ c Department of Frontier Life Sciences, Graduate School of Biomedical Sciences, Nagasaki University, 1-7-1 Sakamoto, Nagasaki, 852-8501, Japan
}

\section{A R T I C L E I N F O}

\section{Article history:}

Received 1 August 2018

Accepted 4 August 2018

Available online 9 August 2018

\section{Keywords:}

Adenosquamous carcinoma

PD-L1

PTEN

Immunohistochemistry

Lung cancer

\begin{abstract}
A B S T R A C T
Background: Lung adenosquamous carcinoma (ASC) is a rare variant of non-small cell lung cancer (NSCLC) with poor prognosis. Certain biological differences may exist between these tumors and other common histological types of NSCLC, including adenocarcinoma (ADC) and squamous cell carcinoma (SCC). The phosphoinositide 3-kinase (PI3K) pathway, which links oncogenes and multiple receptor classes to essential cellular functions, is activated by phosphatase and tensin homolog (PTEN) loss. The PTEN loss has been suggested to induce programmed cell death ligand 1 (PD-L1) expression in various cancer types.

Objective: Here, we sought to determine the relationships between the expression of PTEN and PD-L1 in each component of ASC with ADC and SCC, and clinical parameters.

Material and methods: Tissue microarrays of 148 cases of surgically resected lung ADC and 102 cases of SCC, as well as full sections from 28 ASC cases, were analyzed immunohistochemically for the expression of PTEN and PD-L1.

Results: PD-L1 expression was similar between the adenocarcinoma component of ASC vs. lung ADC and between the squamous component of ASC vs. lung SCC. PTEN loss was higher in lung ADC than in the adenocarcinoma component of ASC and significantly higher in lung SCC than in the squamous component of ASC. PD-L1 expression was higher in the squamous component than in the glandular component of the 28 ASC cases, but PTEN loss was similar. Overall, PTEN loss was higher in lung SCC than in lung ADC and both components of ASC. In lung SCC and glandular portions of ASC, PD-L1 expression levels were significantly associated with those of PTEN. The loss of PTEN correlated with smoking status in patients with lung ADC.

Conclusions: Our results implied that both squamous and glandular components of ASC may share the same oncogenic driver pathway for carcinogenesis. However, the squamous cell components of ASC likely escape the immune surveillance better than the glandular components due to higher PD-L1 expression.
\end{abstract}

(c) 2018 Elsevier Inc. All rights reserved.
Abbreviations: ASC, adenosquamous carcinoma; ADC, adenocarcinoma; SCC, squamous cell carcinoma; NSCLC, non-small cell lung cancer; PI3K, phosphoinositide 3-kinase; PTEN, phosphatase and tensin homolog; PD-L1, programmed cell death ligand 1.

* Corresponding author. Department of Pathology, Nagasaki University Graduate School of Biomedical Sciences, Division of Cancer genomics, Genomic Medical center, Nagasaki University Hospital, 1-7-1 Sakamoto, Nagasaki, 852-8501, Japan.

E-mail address: bfurusato@nagasaki-u.ac.jp (B. Furusato).

\section{Introduction}

Lung cancer is the second most commonly diagnosed cancer, and 234,030 new cases of lung cancer are estimated to be diagnosed in the US in 2018 [1]. Numerous studies demonstrate that tyrosine kinase activation contributes to tumor growth and malignant progression in different tissues, including lung cancer [2,3].

The phosphoinositide 3-kinase (PI3K) signaling axis plays important regulatory roles in cell proliferation and differentiation by activating several oncogenes and downstream effector pathways 
causing tumorigenesis. It can be activated by the loss of phosphatase and tensin homolog (PTEN), mutations in the genes encoding PI3K, or constitutive activation of upstream regulatory receptor tyrosine kinase pathways [4,5]. Hyperactivation of the PI3K pathway might be involved in lung cancer, and certain expression patterns of PTEN confer prognostic significance [6,7].

Interactions between programmed cell death 1 (PD1) and its ligand (PD-L1) constitute a key immune checkpoint, which maintain self-tolerance and protect peripheral tissues by modulating immune responses [8]. Ligation of PD-L1 on cancer cells to PD1 on T cells suppresses $\mathrm{T}$ cell activation and proliferation, inducing $\mathrm{T}$ cell apoptosis [9].

Interconnections between oncogenic driver and immunoregulatory pathways in cancer cells have not been fully elucidated, however, there is evidence for a genetic regulation of immune response [10,11]. PTEN can directly regulate interferon (IFN) response signaling pathways and thus, plays a role in immune regulation [12].

In an in vitro model of NSCLC, PD-L1 expression correlated with reduced PTEN protein expression [13]. In glioblastoma, it was suggested that PTEN loss was an important mechanism for PD-L1 expression in cell lines and tumor specimens [14]. Similar results were found in breast, prostate, and lung carcinomas, indicating that PD-L1 expression is PI3K pathway-dependent [15-17]. PD-L1 protein (but not mRNA) expression was significantly increased in colorectal cancer cells with reduced PTEN expression [18]

Targeted therapy unrelated to genetic alterations is needed for most lung cancers deficient in actionable divergent genes. The application of the newly-developed immune checkpoint inhibitors has been associated with significant response rates in several tumor types [19]. Additionally, anti-PD1/PD-L1-based monoclonal antibodies have shown higher efficacy as a second-line treatment for patients with advanced NSCLC [20]. Hence, PD1 and PD-L1 are potential targets in lung cancers, and PD-L1 expression may be a biomarker for the therapeutic response to anti-PD1/PD-L1 antibodies [21].

Not all tumor cells express PD-L1. In different tumors, many extrinsic and intrinsic factors regulate PD-L1 expression. One such cellular control mechanism includes the inactivation of the tumor suppressor gene PTEN [22,23]. Moreover, resistance to some immune checkpoint inhibitors is partly related to PTEN loss [11,24].

Lung adenosquamous carcinoma (ASC) is an unusual subtype of non-small-cell lung cancer (NSCLC) with worse prognosis than adenocarcinoma (ADC) or squamous cell carcinoma (SCC), and is more aggressive [25]. This observation indicates the existence of certain biological differences among these three histological types of NSCLC [26].

There is no adequate treatment for ASC as deep understanding of its genetic basis and molecular characteristics is lacking. Surgery, platinum-based chemotherapy, and radiotherapy are the primary treatments for ASC. The poor prognosis of ASC patients has prompted a search for more effective treatments, such as targeted therapy. Mutations of the epidermal growth factor receptor (EGFR) gene have been detected in ASC of the lung [27], and recent clinical data have shown that EGFR tyrosine kinase inhibitors are an effective treatment for such cancers [28]. There has also been a case report of a Japanese patient harboring EGFR-activating mutations in ASC after 3 years on gefitinib [29].

Genetic profiling and major driver oncogene alterations in ASC have been detected by different methods. ASC with EGFR mutations is the major type of lung ASC. Mutations of the PI3K signaling pathway genes PIK3CA and PTEN were also found in some cases [30].

A comparative study of PD-L1 expression was performed in SCC and ADC components of ASC and in pure SCC and ADC forms. PD-L1 expression level was similar in the squamous cell component of ASC vs. lung SCC, as well as in the adenocarcinoma component of ASC vs. lung ADC. However, for the first time, it was reported that PD-L1 expression levels differed significantly between the two components of lung ASC [31].

Nonetheless, little is known about the relationship between the expression patterns of PD-L1 and PTEN in ASC. To better understand the biological mechanism underlying the immune and oncogenic nature of ASC, we investigated PD-L1 and PTEN expression levels in ASC, ADC, and SCC, using clinicopathological data.

\section{Materials and methods}

\subsection{Study samples}

We collected 50 histologically proven cases of ASC, 167 cases of ADC, and 116 cases of ASC for this study, which was approved by the Institutional Ethics Committee. The clinicopathological information related to age, sex, smoking history, staging, lymphatic, vascular, and pleural invasion, lymph node metastasis and intrapulmonary metastasis was collected from the clinical records.

Some specimens were excluded because of the absence of tumor cells, presence of questionable inflammatory cells, or edge artifacts in immunohistochemistry (IHC). Therefore, for PD-L1 IHC staining, 28 cases of ASC, 133 cases of ADC, and 88 cases of SCC were included. For PTEN IHC staining, 27 cases of ASC, 148 cases of ADC and 102 cases of SCC were scored.

\subsection{Slide preparation}

For ASC, after preparing full-section hematoxylin and eosinstained slides from formalin-fixed, paraffin-embedded tissue blocks, each case was reviewed for both squamous and glandular components in at least $10 \%$ of the tumor area. These cases were confirmed by a pulmonary pathologist (J.F.) after histological diagnosis based on the World Health Organization criteria [32]. In cases of histologically atypical adenocarcinoma, IHC analyses for napsin $\mathrm{A}$, thyroid transcription factor 1 , and the atypical squamous components p40 and CK14 were also employed to confirm the final results. For ADC and SCC, tissue microarray slides containing 0.7$\mathrm{mm}$ cores of lung ADC and SCC cases were used [33].

\subsection{IHC analyses and antibody selection}

Immunohistochemical analyses of $4-\mu \mathrm{m}$ thick tissue sections stained with anti-PD-L1 monoclonal antibody (clone 28-8) and an anti-PTEN antibody (clone D4.3) were carried out using Ventana Bench Mark XT Automated stainer (Ventana Medical Systems, Tucson, AZ, USA) and BOND III fully automated stainer (Leica Biosystems, Melbourne, Australia) following standard protocols.

\subsection{Scoring and interpretation of IHC}

All stained slides were scanned at $20 \times$ magnification. The scanned slides were scored by two pathologists (A.M.H. and Y.K.). For PTEN, scoring was based on the intensity and distribution of staining in slide images. The intensity of staining was scored as 0 (no staining), 1 (pale yellow), 2 (clay-colored), or 3 (sepia). The distribution was scored as 0 (0-10\%), 1 (11-25\%), 2 (26-50\%), or 3 ( $>50 \%)$. Next, the sum of the intensity score and distribution score was used to generate the total score (TS) of TS0 (sum $\leq 2$ ), TS1 $($ sum $=3)$, and TS2 ( sum $\geq 4)$. TS0 and TS1 were defined as negative $(-)$ expression, and TS2 was defined as positive $(+)$ expression. The expression levels were considered as membrane or cytoplasmic 
components.

PD-L1 expression was scored as the percentage of viable tumor cells exhibiting a complete circumferential or partial linear plasma membrane staining at any intensity in the entire specimen. The percentages were categorized as $<1 \%,<10 \%,<25 \%,<50 \%$ and $\geq 50 \%$. The total expression in less than $1 \%$ or in less than $10 \%$ of all viable tumor cells was regarded as negative $(-)$ expression. The remaining categories were scored as positive $(+)$ expression. The staining of immune cells for PD-L1 was not considered as positive and served only as an internal positive control.

\subsection{Statistical analyses}

Chi-squared and Fisher's contingency tests were used to examine the relationships between the variables. If the $P$ value was less than 0.05 , the result was considered statistically significant. The SAS software was used for all data analyses.

\section{Results}

In ASC, the expression levels of PD-L1 and PTEN in the glandular and squamous regions were examined separately (Fig. 1).

\subsection{PD-L1 expression levels in lung ASC, ADC, and SCC}

When glandular and squamous components of 28 lung ASC samples were examined separately, the levels of PD-L1 expression were $21.43 \%(n=6 / 28)$ and $39.29 \%(n=11 / 28)$, respectively. The difference was nominal and did not reach statistical significance $(P=0.162)$. In lung ADC, PD-L1 expression level was $29.32 \%(n=39 /$ 133), which was similar to that in the glandular component of lung ASC $(P=0.491$; Table 1$)$. In lung SCC, PD-L1 expression level was $30.68 \%$ ( $n=27 / 88)$, which did not differ from that in the squamous component of lung ASC $(P=0.489$; Table 1$)$.

\subsection{PTEN expression levels in lung ASC, $A D C$, and SCC}

The analysis of 27 lung ASC cases revealed that the rate of PTEN loss was similar $(P=>0.99)$ between the glandular component, (48.14\%, $n=13 / 27$ ) and the squamous component $(44.44 \%, n=12$ / $27)$. In lung ADC, the rate of PTEN loss $(63.51 \%, n=94 / 148)$ exceeded the one in the glandular component of lung ASC $(P=0.140$; Table 2). In lung SCC, the rate of PTEN loss was $75.49 \%(n=77 / 102)$, which was significantly higher than that of the squamous component of lung ASC $(P=0.004$; Table 2$)$.

\subsection{PTEN expression and PD-L1 status}

In SCC, PTEN expression was significant in 13 (50\%) of 26 PD-L1positive cases $(P=0.005)$. Only 12 cases out of $60 \mathrm{PD}-\mathrm{L} 1$-negative cases (20\%) showed PTEN expression, while the remaining 48 cases (80\%) were PTEN-negative (Table 3$)$.
Table 1

Comparison of PD-L1 expression between ADC and glandular component of ASC, and between SCC and squamous component of ASC.

\begin{tabular}{llllll}
\hline Types of tumor & \multicolumn{2}{l}{ PD-L1 } & Total & Expression Rate & $P$ \\
\cline { 2 - 4 } & $(+)$ & $(-)$ & & & \multirow{2}{*}{0.491} \\
\hline ADC & 39 & 94 & 133 & $29.32 \%$ & 0.489 \\
Glandular component of ASC & 6 & 22 & 28 & $21.43 \%$ & \\
SCC & 27 & 61 & 88 & $30.68 \%$ & \\
Squamous component of ASC & 11 & 17 & 28 & $39.29 \%$ & \\
\hline
\end{tabular}

ADC, adenocarcinoma; ASC, adenosquamous cell carcinoma; PD-L1, programmed cell death ligand 1; SCC, squamous cell carcinoma.

Both PTEN and PD-L1 expression levels were significantly associated with the glandular component of ASC $(P=0.041)$ (Table 3). There were no statistically significant associations between PTEN and PD-L1 expression levels in the ADC and squamous components of lung ASC $(P=0.558$ and $P=>0.99)$ (Table 3$)$.

\subsection{Relationship between PD-L1 and PTEN expression levels and clinicopathological characteristics}

Among the 28 patients with lung ASC, 23 were men, five were women, eight were smokers, four were nonsmokers, and 16 provided no data. The median age at diagnosis was 69.5 years (range: 49-80 years). The tumors were classified as stage I, II, III, and IV in 10 (35.72\%), eight (28.57\%), three (10.71\%), and one (3.57\%) case, respectively. No data for staging were available in six (21.43\%) cases. Pleural, lymphatic, and vascular invasion, positive node and intrapulmonary metastases were observed in 16 (57.14\%), 21 (74\%), 20 (71.44\%), 10 (35.71\%), and 3 (10.71\%) cases, respectively. Neither PDL1 nor PTEN expression levels in the glandular and squamous components showed a significant association with clinicopathological characteristics (Supplementary Table S1).

Among the 148 patients with lung ADCs, 88 were men, 60 were women, 72 were smokers, and 76 were nonsmokers. The median age at diagnosis was 67 years (range: $30-89$ years). The tumors were classified as stage I, II, III, and IV in 87 (58.78\%), 25 (16.89\%), 28 (18.92\%), and eight (5.41\%) cases, respectively. Pleural, lymphatic, and vascular invasion, positive node and intrapulmonary metastases were observed in 57 (38.51\%), 76 (51.35\%), 56 (37.84\%), 45 (30.41\%), and 26 (17.57\%) cases, respectively. PD-L1 and PTEN expression levels showed no association with most of the clinicopathological characteristics (Supplementary Table S2): only smoking status correlated significantly with PTEN loss $(P=0.017)$ (Table 4).

Among the 102 SCC patients, 96 were men, six were women, 45 were smokers, 38 were nonsmokers, and 19 provided no data. The median age at diagnosis was 67 years (range: $40-88$ years). The tumors were classified as stage I, II, III, and IV in 49 (48.04\%), 34 (33.33\%), 16 (15.69\%), and one (0.98\%) case, respectively. No data for staging were available in two (1.96\%) cases. Pleural, lymphatic, and

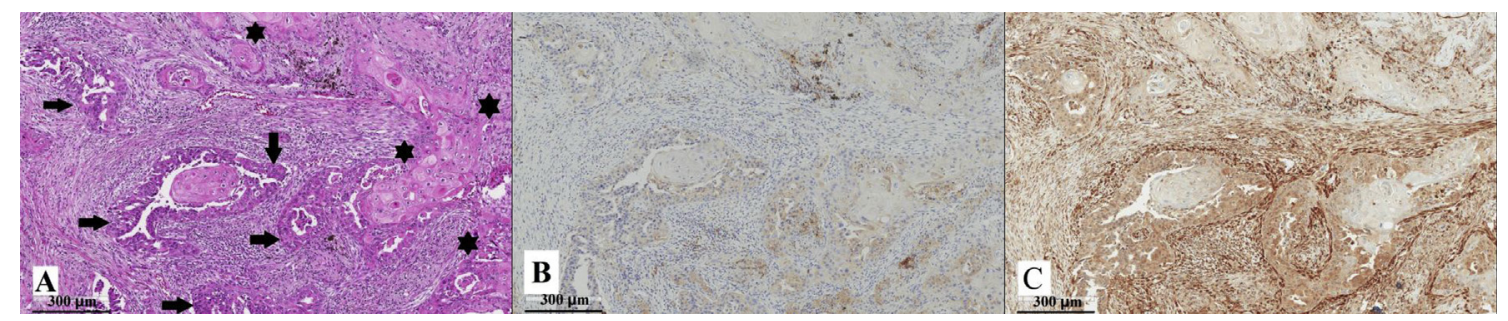

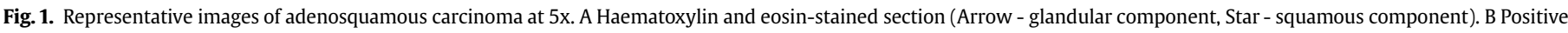
PD-L1 expression in both glandular and squamous components. C Positive PTEN expression in both glandular and squamous components. 
Table 2

Comparison of PTEN expression between ADC and glandular component of ASC, and between SCC and squamous component of ASC.

\begin{tabular}{|c|c|c|c|c|c|}
\hline \multirow[t]{2}{*}{ Types of tumor } & \multicolumn{2}{|c|}{ PTEN } & \multirow[t]{2}{*}{ Total } & \multirow[t]{2}{*}{ Loss of expression rate } & \multirow[t]{2}{*}{$P$} \\
\hline & $(+)$ & $(-)$ & & & \\
\hline $\mathrm{ADC}$ & 54 & 94 & 148 & $63.51 \%$ & 0.14 \\
\hline Glandular component of ASC & 14 & 13 & 27 & $48.14 \%$ & \\
\hline SCC & 25 & 77 & 102 & $75.49 \%$ & 0.004 \\
\hline Squamous component of ASC & 15 & 12 & 27 & $44.44 \%$ & \\
\hline
\end{tabular}

ADC, adenocarcinoma; ASC, adenosquamous cell carcinoma; PTEN, phosphatase and tensin homolog; SCC, squamous cell carcinoma.

Table 3

Comparison of PD-L1 and PTEN expression in ADC, ASC, and SCC.

\begin{tabular}{|c|c|c|c|c|c|c|c|c|}
\hline & \multicolumn{2}{|l|}{ ADC } & \multicolumn{2}{|c|}{ Glandular component of ASC } & \multicolumn{2}{|c|}{ Squamous component of ASC } & \multicolumn{2}{|l|}{ SCC } \\
\hline & \multicolumn{2}{|c|}{$(n=127)(P=\mathrm{NS})$} & \multicolumn{2}{|c|}{$(n=27)(P=0.041)$} & \multicolumn{2}{|c|}{$(n=27)(P=\mathrm{NS})$} & \multicolumn{2}{|c|}{$(\mathrm{n}=86)(P=0.005)$} \\
\hline & $\operatorname{PTEN}(+)$ & $\operatorname{PTEN}(-)$ & $\operatorname{PTEN}(+)$ & $\operatorname{PTEN}(-)$ & $\operatorname{PTEN}(+)$ & $\operatorname{PTEN}(-)$ & $\operatorname{PTEN}(+)$ & $\operatorname{PTEN}(-)$ \\
\hline PD-L1(+) & 17 & 22 & 5 & 0 & 6 & 4 & 13 & 13 \\
\hline PD-L1(-) & 33 & 55 & 9 & 13 & 9 & 8 & 12 & 48 \\
\hline Total & 50 & 77 & 14 & 13 & 15 & 12 & 25 & 61 \\
\hline
\end{tabular}

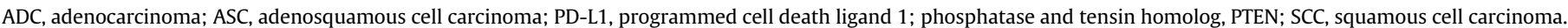

Table 4

Relationship between PTEN expression and smoking status in patients with ADC, each component of ASC and SCC.

\begin{tabular}{|c|c|c|c|c|c|c|c|c|c|}
\hline & & \multicolumn{8}{|c|}{ PTEN expression } \\
\hline & & \multirow{2}{*}{\multicolumn{2}{|c|}{$\begin{array}{l}\text { ADC } \\
(P=0.017)\end{array}$}} & \multirow{2}{*}{\multicolumn{2}{|c|}{$\begin{array}{l}\text { Glandular part of } \\
\text { ASC }(P=0.545)\end{array}$}} & \multirow{2}{*}{\multicolumn{2}{|c|}{$\begin{array}{l}\text { Squamous part of } \\
\text { ASC }(P=>0.99)\end{array}$}} & \multirow{2}{*}{\multicolumn{2}{|c|}{$\begin{array}{l}\text { SCC } \\
(P=0.798)\end{array}$}} \\
\hline & & & & & & & & & \\
\hline & & + & - & + & - & + & - & + & - \\
\hline \multirow[t]{3}{*}{ Smoking } & Present & 19 & 53 & 3 & 4 & 3 & 4 & 10 & 35 \\
\hline & Absent & 35 & 41 & 3 & 1 & 2 & 2 & 10 & 28 \\
\hline & No data & - & - & 8 & 8 & 10 & 6 & 5 & 14 \\
\hline Total & & 54 & 94 & 14 & 13 & 15 & 12 & 25 & 77 \\
\hline
\end{tabular}

PTEN, phosphatase and tensin homolog; ADC, adenocarcinoma; ASC, adenosquamous cell carcinoma; SCC, squamous cell carcinoma.

vascular invasion, positive node and intrapulmonary metastasis were observed in 36 (35.29\%), 38 (37.26\%), 45 (44.12\%), 36 (35.29\%), and 19 (18.63\%) cases, respectively. There was no significant association between PD-L1 and PTEN expression levels and clinicopathological characteristics in SCC cohort (Supplementary Table S3).

\section{Discussion}

The molecular basis of lung cancer is complex and heterogeneous, as it involves the activation of growth-promoting pathways and inhibition of tumor-suppressor pathways [34]. PTEN, a tumor suppressor protein, regulates many cellular processes [35]. In addition to oncogenic pathways, cancer cells use various strategies to escape from immune surveillance [36]. The interaction between PD-L1 and its co-stimulatory signal PD1 induces T cell apoptosis or exhaustion [37]. The relationships between the oncogenic tumor driver pathways and immunoregulatory pathways such as PD-L1 have not been fully elucidated. However, the function of PTEN in the regulation of innate and adaptive immunity has been demonstrated [12]. Thus, we analyzed the expression of PTEN and PD-L1 in ASC and compared their levels to those in more common subtypes of NSCLC.

PD-L1 expression levels were similar between the squamous cell component of ASC and lung SCC (39.29\% vs. 30.68\%), as well as between the glandular component of ASC and lung ADC (21.43\% vs. 29.32\%). Therefore, no trend was apparent for differential PD-L1 expression between SCC or ADC and ASC. This finding also suggests that anti-PD-1/PD-L1 monoclonal antibodies are a promising therapeutic strategy for ASC patients with PD-L1 upregulation.

Our results show that PTEN loss is greater in lung SCC than in lung ADC (75.49\% vs. 63.51\%). This observation is in agreement with previous observations that low PTEN expression is more prevalent in SCC than in ADC $[38,39]$. Furthermore, PTEN loss was more significant in SCC than in the squamous component of ASC. Therefore, the biology of lung ASC may be more complex and divergent than that of SCC and ADC.

It has been suggested that lung ADC can progressively transdifferentiate to SCC with pathologically mixed ASC representing the intermediate stage, which might confer drug resistance and worse prognosis [40]. We found higher PD-L1 expression level in the squamous cell component than in the glandular component of ASC (39.29\% vs. $21.43 \%$ ). Consistently, Shi et al. [31] reported positive PD-L1 expression in $38.89 \%$ and $11.11 \%$ cells of squamous and adenocarcinoma component, respectively, and attributed poorer prognosis of ASC to the escape from immune response resulting from the gain of PD-L1 expression during transdifferentiation.

The results from the screening of the major driver oncogene alterations in lung ASC showed high convergence rates for identical $E G F R$, $K$-ras, and $B$-Raf mutations in both adenomatous and squamous components, suggesting a role for monoclonality in the histogenesis of ASC [41]. In our study, PTEN loss was evident in both ASC components, and the loss rate was virtually the same $(44.44 \%$ vs. $48.14 \%$ ), supporting the monoclonal theory of ASC emergence. Thus, both components may share the same oncogenic driver pathway for carcinogenesis.

In tumor cells, two general mechanisms regulate PD-L1 expression: innate immune resistance and adaptive immune 
resistance. In innate immune resistance, $\mathrm{PD}-\mathrm{L} 1$ expression is driven by constitutive oncogenic signaling pathways in the tumor cell. The alternative mechanism for PD-L1 upregulation in tumors is their adaptation to endogenous tumor-specific immune responses to IFNs, predominantly IFN- $\gamma$, in a process termed adaptive immune resistance [42]. Regarding lung SCC samples in our study, PD-L1 expression significantly correlated with PTEN expression. Eighty percent of PD-L1-negative cases showed PTEN loss $(P=0.005)$. Thus, upregulation of PD-L1 in these PD-L1-positive tumor cells may occur via adaptive immune resistance. It may be assumed that PTEN-positive tumor cells evade antitumor immunity by upregulating the expression of PD-L1. It may also be deduced that lung SCC characterized by the loss of the tumor suppressor PTEN can proceed to tumorigenesis without PD-L1 expression.

Additionally, we found that smoking significantly correlated with PTEN loss in ADC. It is possible that smoking influences the expression of PTEN gene to a certain extent. Indeed; one study concluded that cigarette smoke induced alterations in the PI3K/ PTEN balance in human airway epithelial cells [43].

A limitation of our study is that we evaluated only PTEN expression but not that of other molecular oncogene markers of PI3K and downstream pathways involved in tumor formation. Furthermore, we did not examine factors influencing PD-L1 expression in tumor cells. In addition, we did not study tumorinfiltrating cells, believed to control the response to anti-PD-L1 therapy. Although we used whole tissue sections for ASC, we had only 28 samples, given the rarity of the tumor. Although we sought to retrieve as much ASC clinicopathological data as possible, some data were missing from the electronic records of old cases.

In conclusion, our study provides an insight into the biological mechanisms underlying the immune and oncogenic nature of adenosquamous cell carcinoma. Further multi-institutional studies are required to reproduce these findings and establish their biological and clinical importance.

\section{Funding}

Nagasaki University Priority Research Subject Project Based on Medium-term Goals and Plans.

\section{Disclosure of potential conflicts of interest}

The authors declare no potential conflicts of interest.

\section{Acknowledgments}

The authors are sincerely grateful to Professor Hiroaki Ikeda, Department of Oncology, Nagasaki University Graduate School of Biomedical Sciences, Professor Kazuto Ashizawa, Unit of Translational Medicine, Department of Clinical Oncology, Nagasaki University Graduate School of Biomedical Sciences, Associate professor Chizu Fukushima, Clinical Research Center, Nagasaki University Hospital for supporting research by the Niagara University Priority Research Subject Project Based on Medium-term Goals and Plans fund.

The authors are sincerely grateful to Yuki Imaoka, Department of Pathology, Nagasaki University Graduate School of Biomedical Sciences for their technical assistances.

\section{Appendix A. Supplementary data}

Supplementary data related to this article can be found at https://doi.org/10.1016/j.bbrc.2018.08.037.

\section{Transparency document}

Transparency document related to this article can be found online at https://doi.org/10.1016/j.bbrc.2018.08.037.

\section{References}

[1] W. Street, Cancer Facts \& Figures 2018, 1930, p. 76.

[2] K. Ohtsuka, H. Ohnishi, M. Fujiwara, T. Kishino, S. Matsushima, G. Furuyashiki, H. Takei, Y. Koshiishi, T. Goya, T. Watanabe, Abnormalities of epidermal growth factor receptor in lung squamous-cell carcinomas, adenosquamous carcinomas, and large-cell carcinomas: tyrosine kinase domain mutations are not rare in tumors with an adenocarcinoma component, Cancer 109 (2007) $741-750$.

[3] G. Vlahovic, J. Crawford, Activation of tyrosine kinases in cancer, Oncology 8 (2003) 531-538.

[4] P. Liu, H. Cheng, T.M. Roberts, J.J. Zhao, Targeting the phosphoinositide 3kinase pathway in cancer, Nat. Rev. Drug Discov. 8 (2009) 627-644.

[5] M.D. Siegelin, A.C. Borczuk, Epidermal growth factor receptor mutations in lung adenocarcinoma, Lab. Invest. 94 (2014) 129-137.

[6] E. Sarris, M. Saif, K. Syrigos, The biological role of PI3K pathway in lung cancer, Pharmaceuticals 5 (2012) 1236-1264.

[7] J. Xiao, C.P. Hu, B.X. He, X. Chen, X.X. Lu, M.X. Xie, W. Li, S.Y. He, S.J. You, Q. Chen, PTEN expression is a prognostic marker for patients with non-small cell lung cancer: a systematic review and meta-analysis of the literature, Oncotarget 7 (2016) 57832-57840.

[8] M.E. Keir, M.J. Butte, G.J. Freeman, A.H. Sharpe, PD-1 and its ligands in tolerance and immunity, Annu. Rev. Immunol. 26 (2008) 677-704.

[9] J. He, Y. Hu, M. Hu, B. Li, Development of PD-1/PD-L1 pathway in tumor immune microenvironment and treatment for non-small cell lung cancer, Sci. Rep. 5 (2015), 13110.

[10] R.V. Parry, J.M. Chemnitz, K.A. Frauwirth, A.R. Lanfranco, I. Braunstein, S.V. Kobayashi, P.S. Linsley, C.B. Thompson, J.L. Riley, CTLA-4 and PD-1 receptors inhibit T-cell activation by distinct mechanisms, Mol. Cell Biol. 25 (2005) 9543-9553.

[11] W. Peng, J.Q. Chen, C. Liu, S. Malu, C. Creasy, M.T. Tetzlaff, C. Xu, J.A. McKenzie, C. Zhang, X. Liang, L.J. Williams, W. Deng, G. Chen, R. Mbofung, A.J. Lazar, C.A. Torres-Cabala, Z.A. Cooper, P.L. Chen, T.N. Tieu, S. Spranger, X. Yu, C. Bernatchez, M.A. Forget, C. Haymaker, R. Amaria, J.L. McQuade, I.C. Glitza, T. Cascone, H.S. Li, L.N. Kwong, T.P. Heffernan, J. Hu, R.L. Bassett, M.W. Bosenberg, S.E. Woodman, W.W. Overwijk, G. Lizee, J. Roszik, T.F. Gajewski, J.A. Wargo, J.E. Gershenwald, L. Radvanyi, M.A. Davies, P. Hwu, Loss of PTEN promotes resistance to T cell-mediated immunotherapy, Canc. Discov. 6 (2016) 202-216.

[12] L. Chen, D. Guo, The functions of tumor suppressor PTEN in innate and adaptive immunity, Cell. Mol. Immunol. 14 (2017) 581-589.

[13] J. Wan, X. Ling, B. Peng, G. Ding, miR-142-5p regulates CD4+ T cells in human non-small cell lung cancer through PD-L1 expression via the PTEN pathway, Oncol. Rep. 40 (2018) 272-282.

[14] A.T. Parsa, J.S. Waldron, A. Panner, C.A. Crane, I.F. Parney, J.J. Barry, K.E. Cachola, J.C. Murray, T. Tihan, M.C. Jensen, P.S. Mischel, D. Stokoe, R.O. Pieper, Loss of tumor suppressor PTEN function increases B7-H1 expression and immunoresistance in glioma, Nat. Med. 13 (2007) 84-88.

[15] E.A. Akbay, S. Koyama, J. Carretero, A. Altabef, J.H. Tchaicha, C.L. Christensen, O.R. Mikse, A.D. Cherniack, E.M. Beauchamp, T.J. Pugh, M.D. Wilkerson, P.E. Fecci, M. Butaney, J.B. Reibel, M. Soucheray, T.J. Cohoon, P.A. Janne, M. Meyerson, D.N. Hayes, G.I. Shapiro, T. Shimamura, L.M. Sholl, S.J. Rodig, G.J. Freeman, P.S. Hammerman, G. Dranoff, K.K. Wong, Activation of the PD-1 pathway contributes to immune escape in EGFR-driven lung tumors, Canc. Discov. 3 (2013) 1355-1363.

[16] C.A. Crane, A. Panner, J.C. Murray, S.P. Wilson, H. Xu, L. Chen, J.P. Simko, F.M. Waldman, R.O. Pieper, A.T. Parsa, PI(3) kinase is associated with a mechanism of immunoresistance in breast and prostate cancer, Oncogene 28 (2009) 306-312.

[17] E.A. Mittendorf, A.V. Philips, F. Meric-Bernstam, N. Qiao, Y. Wu, S. Harrington, X. Su, Y. Wang, A.M. Gonzalez-Angulo, A. Akcakanat, A. Chawla, M. Curran, P. Hwu, P. Sharma, J.K. Litton, J.J. Molldrem, G. Alatrash, PD-L1 expression in triple-negative breast cancer, Cancer Immunol. Res. 2 (2014) 361-370.

[18] M. Song, D. Chen, B. Lu, C. Wang, J. Zhang, L. Huang, X. Wang, C.L. Timmons J. Hu, B. Liu, X. Wu, L. Wang, J. Wang, H. Liu, PTEN loss increases PD-L1 protein expression and affects the correlation between PD-L1 expression and clinical parameters in colorectal cancer, PLoS One 8 (2013) e65821.

[19] S.L. Topalian, C.G. Drake, D.M. Pardoll, Immune Checkpoint Blockade: a common denominator approach to cancer therapy, Canc. Cell 27 (2015) 450-461.

[20] R.S. Herbst, P. Baas, D.W. Kim, E. Felip, J.L. Pérez-Gracia, J.Y. Han, J. Molina J.H. Kim, C.D. Arvis, M.J. Ahn, M. Majem, M.J. Fidler, G. de Castro, M. Garrido, G.M. Lubiniecki, Y. Shentu, E. Im, M. Dolled-Filhart, E.B. Garon, Pembrolizumab versus docetaxel for previously treated, PD-L1-positive, advanced non-smallcell lung cancer (KEYNOTE-010): a randomised controlled trial, Lancet 387 (2016) 1540-1550.

[21] M. Zhang, G. Li, Y. Wang, Y. Wang, S. Zhao, P. Haihong, H. Zhao, Y. Wang, PDL1 expression in lung cancer and its correlation with driver mutations: a 
meta-analysis, Sci. Rep. 7 (2017) 10255.

[22] P. Ritprajak, M. Azuma, Intrinsic and extrinsic control of expression of the immunoregulatory molecule PD-L1 in epithelial cells and squamous cell carcinoma, Oral Oncol. 51 (2015) 221-228.

[23] J. Chen, C.C. Jiang, L. Jin, X.D. Zhang, Regulation of PD-L1: a novel role of prosurvival signalling in cancer, Ann. Oncol. 27 (2016) 409-416.

[24] R.W. Jenkins, D.A. Barbie, K.T. Flaherty, Mechanisms of resistance to immune checkpoint inhibitors, Br. J. Canc. 118 (2018) 9-16.

[25] D.T. Cooke, D.V. Nguyen, Y. Yang, S.L. Chen, C. Yu, R.F. Calhoun, Surviva comparison of adenosquamous, squamous cell, and adenocarcinoma of the lung after lobectomy, Ann. Thorac. Surg. 90 (2010) 943-948.

[26] P.L. Filosso, E. Ruffini, S. Asioli, R. Giobbe, L. Macri, M.C. Bruna, A. Sandri, A. Oliaro, Adenosquamous lung carcinomas: a histologic subtype with poor prognosis, Lung Canc. 74 (2011) 25-29.

[27] S.M. Kang, H.J. Kang, J.H. Shin, H. Kim, D.H. Shin, S.K. Kim, J.H. Kim, K.Y. Chung, S.K. Kim, J. Chang, Identical epidermal growth factor receptor mutations in adenocarcinomatous and squamous cell carcinomatous components of adenosquamous carcinoma of the lung, Cancer 109 (2007) 581-587.

[28] Z. Song, B. Lin, L. Shao, Y. Zhang, Therapeutic efficacy of gefitinib and erlotinib in patients with advanced lung adenosquamous carcinoma, J. Chin. Med. Assoc. 76 (2013) 481-485.

[29] K. Kurishima, G. Ohara, K. Kagohashi, H. Watanabe, N. Takayashiki, A. Ishibashi, H. Satoh, Adenosquamous cell lung cancer successfully treated with gefitinib: a case report, Mol. Clin. Oncol. 2 (2014) 282-284.

[30] E. Vassella, S. Langsch, M.S. Dettmer, C. Schlup, M. Neuenschwander, M. Frattini, M. Gugger, S.C. Schäfer, Molecular profiling of lung adenosquamous carcinoma: hybrid or genuine type? Oncotarget 6 (2015) 23905-23916.

[31] X. Shi, S. Wu, J. Sun, Y. Liu, X. Zeng, Z. Liang, PD-L1 expression in lung adenosquamous carcinomas compared with the more common variants of nonsmall cell lung cancer, Sci. Rep. 7 (2017), 46209.

[32] W.D. Travis, E. Brambilla, A.G. Nicholson, Y. Yatabe, J.H.M. Austin, M.B. Beasley, L.R. Chirieac, S. Dacic, E. Duhig, D.B. Flieder, K. Geisinger, F.R. Hirsch, Y. Ishikawa, K.M. Kerr, M. Noguchi, G. Pelosi, C.A. Powell, M.S. Tsao, I. Wistuba, The 2015 World Health Organization classification of lung tumors, J. Thorac. Oncol. 10 (2015) 1243-1260.
[33] C. Kampf, I. Olsson, U. Ryberg, E. Sjöstedt, F. Pontén, Production of tissue microarrays, immunohistochemistry staining and digitalization within the human protein atlas, J. Visualized Exp. 63 (2012) 3620.

[34] J.E. Larsen, J.D. Minna, Molecular biology of lung cancer: clinical Implications, Clin. Chest Med. 32 (2011) 703-740.

[35] C.A. Worby, J.E. Dixon, PTEN, Annual Review of Biochemistry 83 (2014) 641-669.

[36] Y. Yang, Cancer immunotherapy: harnessing the immune system to battle cancer, J. Clin. Invest. 125 (2015) 3335-3337.

[37] W. Ma, B.M. Gilligan, J. Yuan, T. Li, Current status and perspectives in translational biomarker research for PD-1/PD-L1 immune checkpoint blockade therapy, J. Hematol. Oncol. 9 (2016) 47.

[38] M. Cumberbatch, X. Tang, G. Beran, S, Eckersley, X. Wang, R.P.A. Ellston, S. Dearden, S. Cosulich, P.D. Smith, C. Behrens, E.S. Kim, X. Su, S. Fan, N. Gray, D.P. Blowers, I.I. Wistuba, C. Womack, Identification of a subset of human non-small cell lung cancer patients with high PI3K $\beta$ and low PTEN expression, more prevalent in squamous cell carcinoma, Clin. Canc. Res. 20 (2014) 595-603.

[39] G. Jin, M.J. Kim, H.S. Jeon, J.E. Choi, D.S. Kim, E.B. Lee, S.I. Cha, G.S. Yoon, C.H. Kim, T.H. Jung, J.Y. Park, PTEN mutations and relationship to EGFR, ERBB2 KRAS, and TP53 mutations in non-small cell lung cancers, Lung Canc. 69 (2010) 279-283.

[40] S. Hou, S. Zhou, Z. Oin, L. Yang, X. Han, S. Yao, H. Ji, Evidence, mechanism, and clinical relevance of the transdifferentiation from lung adenocarcinoma to squamous cell carcinoma, Am. J. Pathol. 187 (2017) 954-962.

[41] X. Shi, H. Wu, J. Lu, H. Duan, X. Liu, Z. Liang, Screening for major driver oncogene alterations in adenosquamous lung carcinoma using PCR coupled with next-generation and Sanger sequencing methods, Sci. Rep. 6 (2016), 22297.

[42] D.M. Pardoll, The blockade of immune checkpoints in cancer immunotherapy, Nat. Rev. Canc. 12 (2012) 252-264

[43] K. Yamada, K. Asai, Y. Ohara, Y. Sugiyama, A. Shirai, K. Sato, N. Yamamoto, G. Tamagaki, T. Watanabe, K. Konishi, Y. Tochino, M. Uji, H. Kanazawa, K. Hirata, The effect of smoking on phosphoinositide 3-kinase (PI3K) and phosphatase and tensin homolog deleted from chromosome 10 (PTEN) mRNA expression in human airway epithelial cells, Eur. Respir. J. 44 (2014). P3900. 